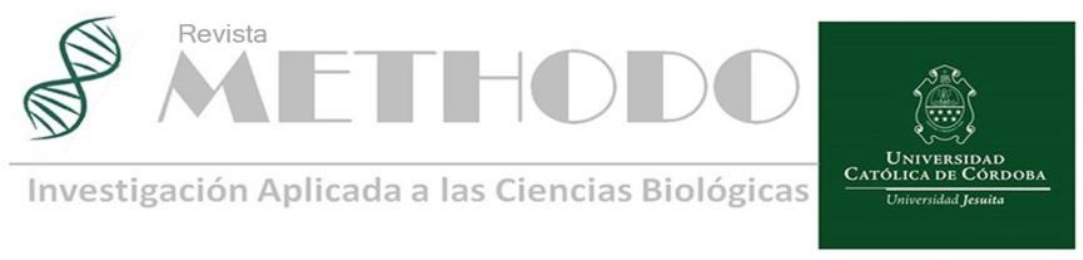

ARTICULO DE REVISION Rev. Methodo 2021;6(1):44-50

https://doi.org/10.22529/me.2021.6(1)07

Recibido 02 Sep. 2020 | Aceptado 27 Oct. 2020 |Publicado 05 Ene. 2021

\title{
La inteligencia artificial en la educación médica y la predicción en salud
}

\section{Artificial intelligence in medical education and health prediction}

\author{
Agustín N. Joison $^{1}$ (iD , Raúl J. Barcudi ${ }^{2}$, Enrique A. Majul ${ }^{3}$, Sergio A. Ruffino ${ }^{3}$, Juan J. De Mateo Rey ${ }^{3}$, \\ Agustin M. Joison ${ }^{4}$, Gustavo Baiardi 1 ${ }^{1,5}$. \\ 1 Universidad Católica de Córdoba, Facultad de Ciencias Químicas. \\ 2 Universidad Católica de Córdoba, Clínica Universitaria Reina Fabiola. Servicio de Cardiología clínica e intervencionista. \\ 3 Universidad Católica de Córdoba. Facultad de Ciencias de la Salud. Clínica Universitaria Reina Fabiola. \\ 4 Globant Empresa de desarrollo de sistemas. Argentina \\ 5 Instituto of investigación Biológica y Tecnológica (IIBYT-CONICET), Universidad Nacional de Córdoba \\ Correspondencia: Agustín Joison, Universidad Católica de Córdoba, Facultad de Ciencias Químicas. Córdoba. Argentina Email: \\ ajoison2001@yahoo.com.ar.
}

\section{Resumen}

La inteligencia artificial tiene el potencial de transformar la forma en que se brinda la atención médica. Puede respaldar mejoras en los resultados y aumentar la productividad y la eficiencia de la prestación de los servicios. En servicios de las diferentes especialidades los avances realizados a nivel hardware deben desarrollarse en paralelo con los métodos de aprendizaje automático, aspectos que la inteligencia artificial contribuye para promover un cambio de paradigma significativo en las más diversas áreas de la medicina. Es importante en la educación médica como eje para el conocimiento y en la toma de decisiones que pueden mejorar el desempeño de los profesionales. Los estudiantes de medicina de nueva generación pueden adaptarse perfectamente a los nuevos métodos digitalizados en un contexto médico globalizado, incluida la inteligencia artificial. Por ello es importante tener como objetivos a implementar en los planes de estudio e introducir programas educativos representativos de esta tecnología. Es fundamental que todas las áreas del Sistema de Salud tengan confianza en los sistemas informáticos específicamente en el aprendizaje profundo, no solo por la información concreta y objetiva que de él se deriva sino también por la posibilidad de predecir eventos futuros, brindando alta certeza en cuanto al diagnóstico y tratamiento de enfermedades.

Palabras claves: Inteligencia artificial, Cardiología, Algoritmos, Red neuronal, salud, predicción.

\begin{abstract}
Artificial intelligence has the potential to transform the way healthcare is delivered. You can support improved results and increase the productivity and efficiency of service delivery. In the services of the different specialties, the advances made at the hardware level must be developed in parallel with the methods of machine learning, aspects that artificial intelligence contributes to promote a significant paradigm shift in the most diverse areas of medicine. It is important in medical education as an axis for knowledge and in making decisions that can improve the performance of professionals. New generation medical students can perfectly adapt to new digitized methods in a globalized medical context, including artificial intelligence. For this reason, it is important to have as objectives to be implemented in the study plans and to introduce educational programs that are representative of this technology. It is essential that all areas of the Health System have confidence in computer systems specifically in deep learning, not only because of the concrete and objective information that is derived from it but also because of the
\end{abstract}


possibility of predicting future events, providing high certainty regarding to the diagnosis and treatment of diseases.

Keywords: Artificial intelligence, Cardiology, Algorithms, Neural network, Health, Prediction

\section{Introducción}

La inteligencia artificial (IA) tiene el potencial de transformar la forma en que se brinda el servicio. Puede respaldar mejoras en los resultados de la misma, aumentar la productividad y la eficiencia de la prestación y permitir que los sistemas de salud brinden más y mejor atención a más personas. La IA puede ayudar a mejorar la experiencia de los profesionales sanitarios, y dedicar más tiempo a la atención directa del paciente, reduciendo el agotamiento. Por último, puede respaldar la prestación más rápida, principalmente al acelerar el tiempo de diagnóstico, y ayudar a los sistemas de salud a mejorar su administración de manera más proactiva, asignando recursos donde puede tener el mayor impacto ${ }^{1}$.

El término "Inteligencia Artificial" (IA) se definió como la ciencia y la ingeniería para fabricar máquinas inteligentes ${ }^{2} \mathrm{El}$ desarrollo de la IA se inició a principios de la década de 1990 como parte de un campo relevante de la informática en busca de encontrar similitudes y características de la inteligencia humana, en cuanto al pensamiento y razonamiento e incluso a ver y como oír ${ }^{3}$. Las primeras aplicaciones de la IA se realizaron analizando datos sanitarios, plazos y opciones de pago, como herramientas innovadoras para gestionar la información del paciente y tomar decisiones. La necesidad de datos y toma de decisiones ha permitido dar respuesta a problemas de salud. Los objetivos de este resumen son para entender la importancia de la aplicación de esta tecnología en el cuidado y tratamiento de la salud, y además destacar la posibilidad en un futuro cercano de incorporarla en la educación médica.

\section{Concepto y aplicación de inteligencia artificial}

La IA facilita una mayor accesibilidad, relevancia y capacidad de acción de la información sanitaria. La IA se ha desarrollado rápidamente en medicina $\mathrm{y}$ atención médica junto con los avances en computación, en el aprendizaje y la disponibilidad de grandes conjuntos de datos (Big Data) de registros médicos electrónicos (RME) ${ }^{4}$. El análisis de datos de salud es un área multidisciplinar relacionada con el aprendizaje automático y el reconocimiento de patrones, el control inteligente de enfermedades y la prevención para el cuidado de la salud. El objetivo básico de la enfermedad basada en la prevención del análisis de datos es tomar datos de pacientes del mundo real y ayudar a reducir el riesgo del paciente ${ }^{5}$.

La IA es una disciplina científica que se enfoca en comprender y crear algoritmos informáticos similares a los que pueden hacer los humanos. La IA está ganando impulso en la atención médica, donde la pregunta es: "¿Pueden pensar las máquinas?" La IA ha recorrido un largo camino. Los ejemplos de avances en IA incluyen procesamiento de lenguaje natural, reconocimiento de voz, agentes virtuales, gestión de decisiones, aprendizaje automático, aprendizaje profundo y automatización de procesos robóticos. La IA implica el desarrollo de algoritmos informáticos para realizar tareas típicamente asociadas con la inteligencia humana ${ }^{6,7}$.

Con la información médica creciendo a una velocidad vertiginosa, los médicos tienen problemas para mantenerse al día. Esto conduce a una sobrecarga de información y crea presión para memorizar todo este contenido para aprobar los exámenes de licencia médica de los Estados Unidos para calificar para puestos de residencia. Los médicos de hoy trabajan más horas y también se espera que brinden atención coordinada en una sociedad que envejece con afecciones complejas y comorbilidades donde los costos de atención médica están aumentando y las regulaciones imponen una carga adicional a los procesos ${ }^{8,9}$.

Los estudios postulan que la posibilidad de que los médicos sean reemplazados por la IA en la atención médica es incorrecta, no se trata necesariamente de enfrentar las mentes humanas contra la máquina, sino de expandir, agudizar y, a veces, calmar la mente del profesional para resolver los problemas que presentan sus pacientes. El papel de la IA en la medicina es desarrollar algoritmos de diagnóstico para robots quirúrgicos ${ }^{10}$.

\section{La inteligencia artificial en áreas específicas de salud}

En el área de cardiología, los avances realizados a nivel de hardware deben desarrollarse en paralelo con los métodos de aprendizaje automático, aspectos que contribuyen a la IA para promover un cambio de paradigma significativo en las más diversas áreas de la medicina. Conocimiento $\mathrm{y}$, 
particularmente en Cardiología, por su capacidad para apoyar la toma de decisiones que pueden mejorar el desempeño diagnóstico y pronóstico. Estos impactos deben ser evaluados para su aplicación en seguridad del paciente, personalización de la atención, valorización del paciente, dentro de un campo de vigilancia tecnológica, que gradualmente consolida la IA como fundamental para una excelente práctica médica $^{11,12}$.

La cardiología necesita inteligencia artificial para el desarrollo de algoritmos de IA y, en consecuencia, no requiere muchas hipótesis en relación con los datos existentes, lo que permite un alto nivel de evidencia debido a su alto rendimiento, lo que sin duda representa un cambio de paradigma significativo en la medicina basada en la evidencia. Cabe señalar que los ensayos clínicos tradicionales son generalmente lentos, costosos, lentos y de tamaño limitado. Además, cuando la base de datos se alimenta con más datos (saludables), en general, se produce una mejora en el rendimiento de los algoritmos, que permiten que los estudios sean continuos en el tiempo ${ }^{13,14}$.

Los cardiólogos aplicaron algoritmos para detectar arritmias a partir del registro de información de un electrocardiograma. Los datos se obtuvieron y analizaron a partir de un árbol de decisiones y reglas basadas en umbrales. Se utilizaron algoritmos genéticos para seleccionar las características más adecuadas para ser utilizadas en el trabajo. Para la detección de fibrilación auricular, se obtuvo una puntuación F1 (media de valor predictivo positivo y sensibilidad) de 0,81 . Otros autores utilizaron el Análisis de Tomografía Espacial Fase Cardíaca, así como la realización de ejercicios o estrés farmacológico, combinados con modelos ML (por ejemplo, algoritmos genéticos) para analizar señales de fase torácica. En este estudio, los autores utilizaron esta herramienta para evaluar a pacientes con enfermedad coronaria y dolor en el pecho que fueron remitidos por el médico para una angiografía. Se estudiaron 606 pacientes y los resultados mostraron $92 \%$ de sensibilidad, $62 \%$ de especificidad y $96 \%$ de valor predictivo de enfermedad coronaria ${ }^{15,16,17,18 .}$

La ecocardiografía es actualmente uno de los métodos de imagen más utilizados en cardiología, la ecografía también tiene ventajas en cuanto a su portabilidad, rapidez y accesibilidad. Pero depende del operador y requiere un largo tiempo de entrenamiento para lograr una interpretación precisa de los datos adquiridos. La IA se utiliza para estandarizar mejor las imágenes ecocardiográficas y reducir la dependencia del operador. Ya ha demostrado la capacidad de ayudar en el análisis de imágenes de eco, lo que permite la generación de importantes variables cardíacas sobre la marcha con la clasificación automatizada de vistas ecocardiográficas ${ }^{19}$.

Una de las patologías con mayor dificultad para decidir sobre el tratamiento y seguimiento es la Diabetes mellitus (DM). La DM se refiere colectivamente a un grupo de enfermedades que resultan de la disfunción del sistema de regulación de la glucosa, y en cada clase de diabetes, se requiere un diagnóstico rápido, educación del paciente en el autocuidado y atención médica continua para prevenir complicaciones agudas como cetoacidosis, enfermedad renal, retinopatía, pie diabético, enfermedad cardiovascular o accidente cerebrovascular. Los algoritmos inteligentes se utilizan ampliamente para el desarrollo de herramientas y aplicaciones que pueden mejorar el manejo efectivo de enfermedades complicadas, incluida la diabetes, por lo que la IA es un papel clave en el reconocimiento de estos sistemas como ayudas terapéuticas de rutina para pacientes con diabetes ${ }^{20}$.

\section{Inteligencia artificial en la decisión médica}

Las decisiones médicas también se referirán a los servicios médicos de urgencias para la gestión del pre-hospitalario en paciente traumático y su traslado al servicio de urgencias en función del estado del mismo. Las decisiones que toman los profesionales para predecir el pronóstico son importantes para la comunicación entre el técnico de RME pre-hospitalario y el departamento de emergencias del hospital para proporcionar instrucciones médicas en línea y prepararse para la gestión hospitalaria. Diferentes estudios demostraron que el uso del algoritmo de IA predijo con precisión cuando y situación el paciente fue trasladado del SEM a cuidados críticos en la unidad hospitalaria ${ }^{21,22,23 .}$

Actualmente en medicina, los procesos de toma de decisiones se basan en la disponibilidad de evidencia objetiva y confiable, la investigación, así como la adecuada interpretación de los hechos disponibles con la incorporación de las relaciones riesgo-beneficio del paciente en cada paso de decisión; sin embargo, la práctica de la medicina en el mundo real nos ha enseñado que la evidencia no siempre está disponible, la penetración del conocimiento lleva tiempo y las decisiones sobre pacientes individuales pueden no siempre ser objetivas $^{24}$.

La mayoría de los cardiólogos de hoy en día tienen más probabilidades de asociar el término IA con un fenómeno de ciencia ficción aún distante, pero la realidad es que estás a punto de conquistar la medicina, incluida la medicina cardiovascular. Dawes y col. publicaron un algoritmo basado en 
imágenes de resonancia magnética cardíaca de patrones tridimensionales de movimiento cardíaco sistólico que les permitió predecir el resultado en pacientes con hipertensión pulmonar con alta precisión. Brevemente, se utilizaron datos médicos de 250 pacientes para el estudio, y el software copió cómo más de 30.000 puntos en sus corazones se contraían con cada latido. Esto creó un corazón virtual tridimensional para cada paciente, mientras que el algoritmo aprendió qué características estaban asociadas con la muerte prematura o la insuficiencia cardíaca derecha ${ }^{25}$.

La IA también puede ayudar a respaldar la investigación de fármacos y mejorar la eficiencia del desarrollo de nuevos fármacos. Finalmente, la combinación de inteligencia artificial y robots quirúrgicos mejorará la precisión de muchas operaciones complejas y difíciles. Con el desarrollo de la inteligencia artificial, el análisis de big data y las tecnologías de computación en la nube, la inteligencia artificial puede brindar a los pacientes servicios médicos de alta calidad ${ }^{26}$.

\section{Inteligencia artificial en estudiantes de medicina}

Los estudiantes de medicina deben aprender como parte de sus conocimientos universitarios la experiencia adecuada en el uso de la IA para aplicarla a sus pacientes en el futuro. Su preparación no es simplemente aprender a usar una tecnología de software, sino un conocimiento suficiente de medicamentos básicos y clínicos (que son los fundamentos de la práctica médica y son clave para comprender cómo usar la IA para la medicina), ciencia de datos, bioestadística y medicina basada en evidencia. Incluso como estudiante de medicina, uno no debería aceptar pasivamente historias relacionadas con la IA médica en los medios de comunicación y en Internet. Los estudiantes de medicina deben tratar de desarrollar habilidades para distinguir la información correcta del bombo y los efectos ${ }^{27}$.

La educación de los estudiantes de medicina implica el aprendizaje durante muchos años desde la formación de pregrado hasta la formación de posgrado como parte de su especialización y más allá, como maestrías, doctorados también conocidos como "educación médica continua". Al principio hubo poco interés en la aplicación de la IA en la educación médica durante la década de 1980, pero más tarde y, curiosamente, se empezaron a publicar trabajos que mostraban la eficacia de la IA en el diagnóstico médico. Esto promovió un aumento en la investigación y el desarrollo de la IA en la educación médica en los últimos años ${ }^{28}$.

Los estudiantes de medicina de nueva generación pueden adaptarse perfectamente a los nuevos métodos digitalizados en un contexto médico globalizado, incluida la IA. Por ello es importante tener como objetivos a implementar en los planes de estudio e introducir programas educativos representativos de esta tecnología ${ }^{29}$. La expansión de la IA en la práctica médica ha generado debates sobre su aplicación en el área de la educación médica en las universidades. En el Reino Unido (UK), el uso de nuevos métodos digitales en salud ha sido objeto de un reciente gobierno nacional ${ }^{30}$. Topol explica con mucho énfasis explícito la importancia de las herramientas digitales novedosas en la enseñanza de pregrado y posgrado con el objetivo de ayudar a las futuras generaciones de estudiantes de medicina en su actividad clínica, incluidos los apoyados por la IA. Actualmente, las facultades de medicina del Reino Unido no tienen este tipo de tecnología incluida en sus planes de estudio, pero hay una recomendación del General Medical Council de que la incorporen en el futuro. Existe alguna evidencia de que ciertas facultades de medicina del Reino Unido optan por ofrecer oportunidades educativas limitadas en el uso de la IA en la salud; sin embargo, existe cierta resistencia a la formación y aprendizaje de dicha tecnología por parte de los propios alumnos y docentes. Además, para involucrar efectivamente a los estudiantes de medicina en este tema, sería útil comprender su percepción de la IA como una cohorte. No es descabellado asumir que el ambiente actual de entusiasmo por la IA influye en la actitud y el comportamiento de los estudiantes de medicina. De relevancia específica para la radiología, esto ha sido estudiado previamente por grupos en Canadá ${ }^{31}$.

Otros estudios revelaron que grupos de estudiantes que hacían radiología no participaban en la aplicación de la IA, porque argumentaban que dicha tecnología los reemplazaría. En cualquier caso, no se les conoce desde un punto de vista profesional, si este es el caso, con respecto a las actitudes de los estudiantes del Reino Unido de que la IA puede ser una amenaza para ellos en el futuro $^{32}$.

\section{Mirando hacia el futuro}

El uso de la IA y el aprendizaje profundo como un nuevo paradigma para la resolución de problemas fue adoptado por la División de Recursos de Investigación del Instituto Nacional de Salud, bajo la dirección de Robert Raub, quien tiene un alumno de William Yamamoto en la Universidad de Pensilvania, con el objetivo de conocer y ampliar el enfoque desde lo estrictamente estadístico hasta el modelado de inferencia del conocimiento fisiológico y clínico subyacente que sustenta la toma de decisiones diagnósticas y terapéuticas ${ }^{33}$. 
Esta revisión tiene como uno de los objetivos comenzar a comprender la posibilidad de utilizar la IA en la educación médica, para que en un futuro se pueda incluir en la evaluación curricular y estudiar si hay una mejora en el aprendizaje de los estudiantes, con investigaciones principalmente sobre el último. Analizar también los desafíos que dificultan la implementación de la IA en la educación médica, los cuales están relacionados con la mejor manera de evaluar la efectividad de la IA y manejar las dificultades técnicas asociadas al desarrollo efectivo y productivo de un sistema de IA.

Esta tecnología, que nació con cierta incertidumbre y calidez de los profesionales para aceptar este paradigma con respecto a la información que brinda, termina como un nuevo paradigma en el cuidado de la salud por la comprensión de los algoritmos y la importancia del aprendizaje automático. Tenemos la esperanza de que dilucidar estas conexiones desmitifique estas técnicas y proporcione un conjunto de expectativas razonables para el papel de la IA en la atención de la salud. Es fundamental que todas las áreas del Sistema de Salud tengan confianza en los algoritmos informáticos específicamente en el aprendizaje profundo, no solo por la información concreta y objetiva que de él se deriva sino también por la posibilidad de predecir eventos futuros, brindando alta certeza en cuanto al diagnóstico y tratamiento de enfermedades (Figura 1).

\section{Conclusiones}

Creemos que la IA tiene un papel importante que desempeñar en la práctica médica del futuro. En forma de aprendizaje automático, es la capacidad principal detrás del desarrollo de la medicina de precisión, ampliamente aceptada como un avance necesario en mejorar la salud. Aunque los primeros esfuerzos para proporcionar recomendaciones de diagnóstico y tratamiento han demostrado ser un desafío, esperamos, que la IA finalmente acompañe la educación médica. También parece cada vez más claro que los sistemas de IA no reemplazarán a los profesionales médicos a gran escala, sino que aumentarán sus esfuerzos para cuidar a los pacientes.

\section{Bibliografía}

1. Jiang F, Jiang Y, Zhi H, Dong Y, Li H, Ma S, et al. Artificial intelligence in healthcare: past, present and future. Stroke Vasc Neurol. 2017;2(4):230-43.

2. Abidi SSR, Abidi SR. Intelligent health data analytics: A convergence of artificial intelligence and big data. Healthc Manage Forum. 2019;32(4):178-82.

3. Asadi H, Dowling R, Yan B, Mitchell P. Machine learning for outcome prediction of acute ischemic stroke post intra-arterial therapy. PLoS One. 2014;9(2):e88225.

4. Caliebe A, Leverkus F, Antes G, Krawczak M. Does big data require a methodological change in medical research? BMC Med Res Methodol. 2019;19(1):125.

5. Razzak MI, Imran M, Xu G. Big data analytics for preventive medicine. Neural Comput Appl. 2019;16:1-35.

6. Paranjape K, Schinkel M, Nannan Panday R, Car J, Nanayakkara P. Introducing Artificial Intelligence Training in Medical Education. JMIR Med Educ. 2019;5(2):e16048.

7. $\mathrm{Yu} \mathrm{KH}$, Beam AL, Kohane IS. Artificial intelligence in healthcare. Nat Biomed Eng. 2018;2(10):719-31.

8. Weaver SJ, Che XX, Petersen LA, Hysong SJ. Unpacking Care Coordination Through a Multiteam System Lens: A Conceptual Framework and Systematic Review. Med Care. 2018;56(3):247-59.

9. Elliott J, Stolee P, Boscart V, Giangregorio L, Heckman G. Coordinating care for older adults in primary care settings: understanding the current context. BMC Fam Pract. 2018;19(1): 137.

10. Oh S, Kim JH, Choi SW, Lee HJ, Hong J, Kwon SH. Physician Confidence in Artificial Intelligence: An Online Mobile Survey. J Med Internet Res. 2019;21(3):e12422.

11. Dilsizian ME, Siegel EL. Machine Meets Biology: a Primer on Artificial Intelligence in Cardiology and Cardiac Imaging. Curr Cardiol Rep. 2018;20(12):139.

12. Chang AC. Artificial intelligence in pediatric cardiology and cardiac surgery: Irrational hype or paradigm shift? Ann Pediatr Cardiol. 2019;12(3):191-4.

13. Johnson KW, Torres Soto J, Glicksberg BS, Shameer K, Miotto R, Ali M, et al. Artificial Intelligence in Cardiology. J Am Coll Cardiol. 2018;71(23):2668-79.

14. Price WN. Big data and black-box medical algorithms. Sci Transl Med. 2018;10(471).

15. Paredes S, Rocha T, de Carvalho P, Henriques J, Morais J, Ferreira J. Integration of Different Risk Assessment Tools to Improve Stratification of Patients with Coronary Artery 
Disease. Med Biol Eng Comput. 2015;53(10):1069-83.

16. Al-Mallah MH, Elshawi R, Ahmed AM, Qureshi WT, Brawner CA, Blaha MJ, et al. Using Machine Learning to Define the Association between Cardiorespiratory Fitness and All-Cause Mortality (from the Henry Ford Exercise Testing Project). Am J Cardiol. 2017;120(11):2078-84.

17. Smisek R, Hejc J, Ronzhina M, Nemcova A, Marsanova L, Kolarova J, et al. Multi-stage SVM approach for cardiac arrhythmias detection in short single-lead ECG recorded by a wearable device. Physiol Meas. 2018;39(9):094003.

18. Stuckey TD, Gammon RS, Goswami R, Depta JP, Steuter JA, Meine FJ, 3rd, et al. Cardiac Phase Space Tomography: A novel method of assessing coronary artery disease utilizing machine learning. PLoS One. 2018;13(8):e0198603.

19. Westcott RJ, Tcheng JE. Artificial Intelligence and Machine Learning in Cardiology. JACC Cardiovasc Interv. 2019;12(14):1312-4.

20. Rigla M, García-Sáez G, Pons B, Hernando ME. Artificial Intelligence Methodologies and Their Application to Diabetes. J Diabetes Sci Technol. 2018;12(2):303-10.

21. Kwon JM, Jeon KH, Kim HM, Kim MJ, Lim $\mathrm{S}$, Kim KH, et al. Deep-learning-based out-ofhospital cardiac arrest prognostic system to predict clinical outcomes. Resuscitation. 2019;139:84-91.

22. Seymour CW, Kahn JM, Cooke CR, Watkins TR, Heckbert SR, Rea TD. Prediction of critical illness during out-of-hospital emergency care. Jama. 2010;304(7):747-54.

23. Smith GB, Prytherch DR, Meredith P, Schmidt PE, Featherstone PI. The ability of the National Early Warning Score (NEWS) to discriminate patients at risk of early cardiac arrest, unanticipated intensive care unit admission, and death. Resuscitation. 2013;84(4):465-70.

24. Otokiti A. Using informatics to improve healthcare quality. Int J Health Care Qual Assur. 2019;32(2):425-30.

25. Dawes TJW, de Marvao A, Shi W, Fletcher T, Watson GMJ, Wharton $\mathrm{J}$, et al. Machine
Learning of Three-dimensional Right Ventricular Motion Enables Outcome Prediction in Pulmonary Hypertension: A Cardiac MR Imaging Study. Radiology. 2017;283(2):381-90.

26. Yan Y, Zhang JW, Zang GY, Pu J. The primary use of artificial intelligence in cardiovascular diseases: what kind of potential role does artificial intelligence play in future medicine? J Geriatr Cardiol. 2019;16(8):585-91.

27. Ochodo EA, de Haan MC, Reitsma JB, Hooft L, Bossuyt PM, Leeflang MM. Overinterpretation and misreporting of diagnostic accuracy studies: evidence of "spin". Radiology. 2013;267(2):581-8.

28. Chan KS, Zary N. Applications and Challenges of Implementing Artificial Intelligence in Medical Education: Integrative Review. JMIR Med Educ. 2019;5(1):e13930.

29. Han ER, Yeo S, Kim MJ, Lee YH, Park KH, Roh $\mathrm{H}$. Medical education trends for future physicians in the era of advanced technology and artificial intelligence: an integrative review. BMC Med Educ. 2019;19(1):460.

30. Sit C, Srinivasan R, Amlani A, Muthuswamy K, Azam A, Monzon L, et al. Attitudes and perceptions of UK medical students towards artificial intelligence and radiology: a multicentre survey. Insights Imaging. 2020;11(1):14.

31. Gong B, Nugent JP, Guest W, Parker W, Chang PJ, Khosa F, et al. Influence of Artificial Intelligence on Canadian Medical Students' Preference for Radiology Specialty: ANational Survey Study. Acad Radiol. 2019;26(4):566-77.

32. Pinto Dos Santos D, Giese D, Brodehl S, Chon $\mathrm{SH}$, Staab W, Kleinert R, et al. Medical students' attitude towards artificial intelligence: a multicentre survey. Eur Radiol. 2019;29(4):1640-6.

33. Amisha, Malik P, Pathania M, Rathaur VK. Overview of artificial intelligence in medicine. J Family Med Prim Care. 2019;8(7):2328-31. 


\section{Anexo figura}

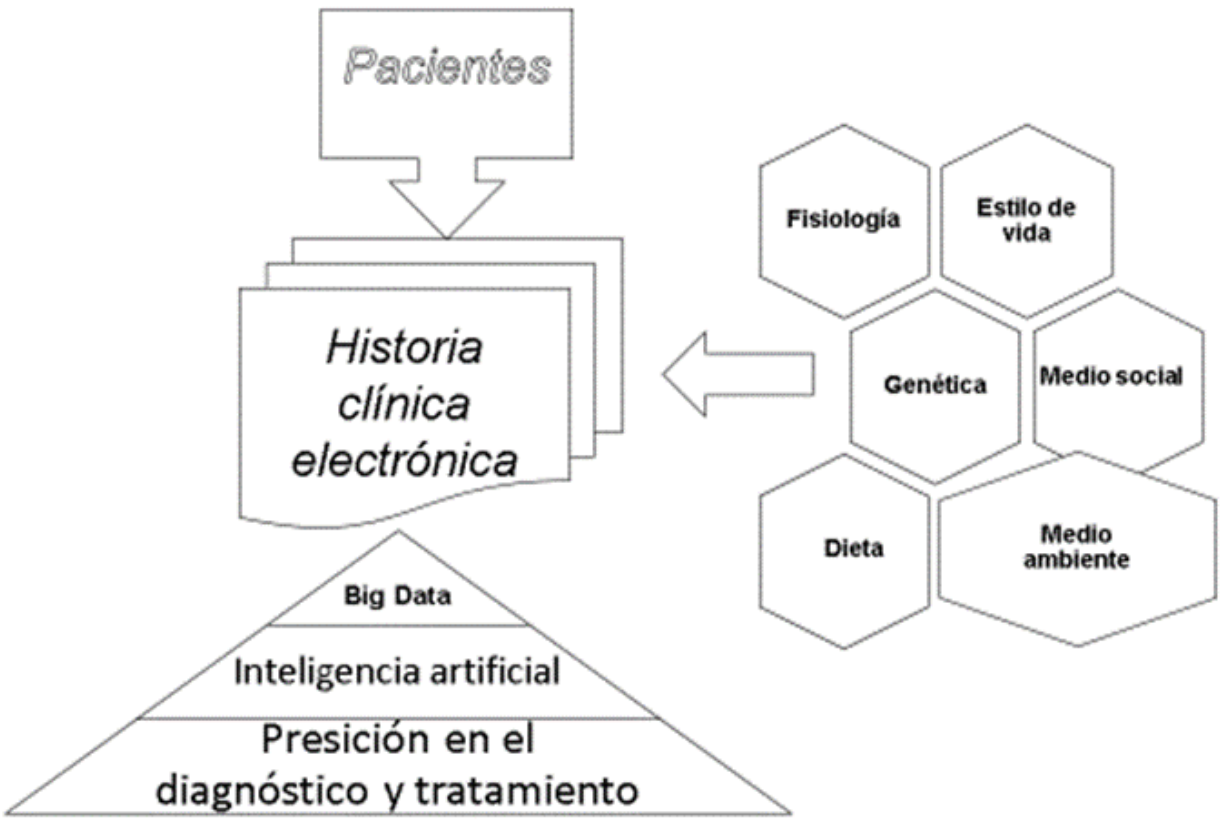

Figura 1. interrelaciones en la enseñanza y aplicación de la medicina de los próximos años.

\section{(c)(1) $(0$}

